\title{
Effectiveness of somatostatin analogs as first-line or second-line treatment in acromegaly: single centre experience
}

Agne Abraitiene MD PhD'.2

Vaidotas Uhanavicius MD PhD

,Vilnius University Faculty of Medicine

2Vilnius University Hospital Santarikių klinikos, Centre of Endocrinology

\section{Introduction:}

The IGF-I ( () . In the majority of patients ( $>95 \%$ ) it results from a GH-secreting pituitary adenoma (1). With the incidence of 3 to 4 new cases per million and the prevalence of 40 to 125 per million, acromegaly is a rare disease (2). Acromegaly affects almost every organ an associated with increased morbidity and mortality and reduced quality of life. Increased mortality is reverted to that of the normal population after normalization of GH and IGF-I levels by successful treatment (3). Transsphenoidal surgery remains the cornerstone of those with macroadenomas (4). Because complete resection is not always feasible, adjunctive therapy is frequently necessary. Sometostatin analogs (SSA) are the most widely used medications to control $G H$ hypersecretion after falure of surgery or as primary medical the SSA treatment.

\section{Patients and methods:}

It was a a trospectiv study conducted in the centre of Endocrinology in Vilnius University hospital Santarikiu klinikos, a tertiary referral centre for endocrinology and diabetes mellitus. Data of 49 patients who were diagnosed, treated or followed-up because of acromegaly between 2007 and 2013 were extracted from the hospital's electronic database. In all cases diagnosis of acromegaly was established on the basis of characteristic clinical features, elevated for age and gender IGF-I, and failure to suppress $\mathrm{GH}<3 \mathrm{mIU/l}$ after $75 \mathrm{~g}$ oral glucose tolerance test (OGTT) or elevated random GH level. Magnetic resonance imaging (MRI) was performed in all patients except one in whom computerized tomography was done because of contraindications to MRI. Tumor size was classified as micro- $(<10 \mathrm{~mm})$ and macroadenoma. The following data were collected from medical records: demographic features, date of diagnosis, tumor size, current medical therapy and whether it was primary therapy, radiotherapy date and method) were also registered.

Disease remission was defined according to the latest guidelines (4): normal age- and gender-adjusted IGF-I, and nadir GH $<3$ mIU after $75 \mathrm{~g}$ OGTT or random $\mathrm{GH}<3 \mathrm{~m} \mathrm{mIUl}$. Partial disease control was documented according local acromegaly management guidelines in cases when IGF-I level dropped, but remained $<30 \%$ above upper limit of normal (adjusted for age and gender), and random GH was below $7.5 \mathrm{mlU} /$. Uncontrolled disease was defined in cases when IGF-I level was elevated $>30 \%$ above age- an gender-adjusted upper limit of normal and random GH was above $7.5 \mathrm{mU} /$. In case of discordant IGF-l and GH results, a patient was assigned to one of study groups according to worse result. Statistical analysis was performed using SPSS (Statistical Package for Social Sciences) 15.0 software.

Table 1. Characteristics of study population Table 2. Treatment modalities and disease outcomes Patient population
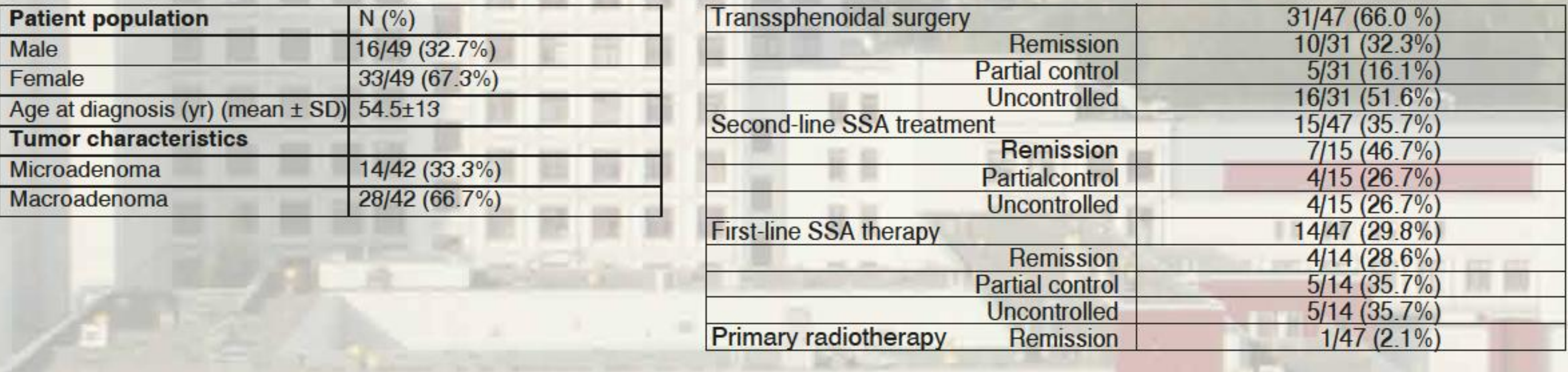

\section{Results}

Demographics and clinical data

49 patients ( 26 newly diagnosed and 23 followed-up or treated) were included in the analysis. Patient population consisted of 16 males and 33 females with a mean age at diagnosis of $54.5 \pm 13$ years. All cases except two (empty sella) were caused by pituitary adenomas, of which $66.7 \%$ were macroadenomas and $33.3 \%$ were microadenomas (table 1). Data about pituitary adenoma preoperative size were no found in the charts in five cases.

Results of treatment

Transsphenoidal operation was applied as the first-line therapy in 31 patients and it led to disease remission in 10 of them (partial disease control was achieved in 5 of operated patients). Primary SSA therapy was administered in 14 of cases due to contraindications or refusal of surgery. One patient was not administered any treatment because of severe comorbidities, one was treated with primary radiotherapy. In two cases data about treatment is not available as the patients are lost to follow-up. O surgically treated patients, 15 received second-line SSA therapy because of uncontrolled disease. Radiotherapy as a third-line treatment was applied in the $5(33 \%)$ patients with a postoperative disease recurrence (table 2). Based on the latest $\mathrm{GH}$ and IGF-1 results, control and partial control were achieved in $4(28.6 \%)$ and $5(35.7 \%)$ patients in first-line SSA therapy group, and in $7(46.7 \%)$ and $4(26.7 \%)$ patients in second-line SSA therapy group. $5(35.7 \%)$ and $4(26.7 \%)$ of patients in first-line and second-line SSA treatment groups remained uncontrolled (figure 1).

Figure 1. Disease outcomes in second-line SSA and first-line SSA treatment groups Second-Line SSA

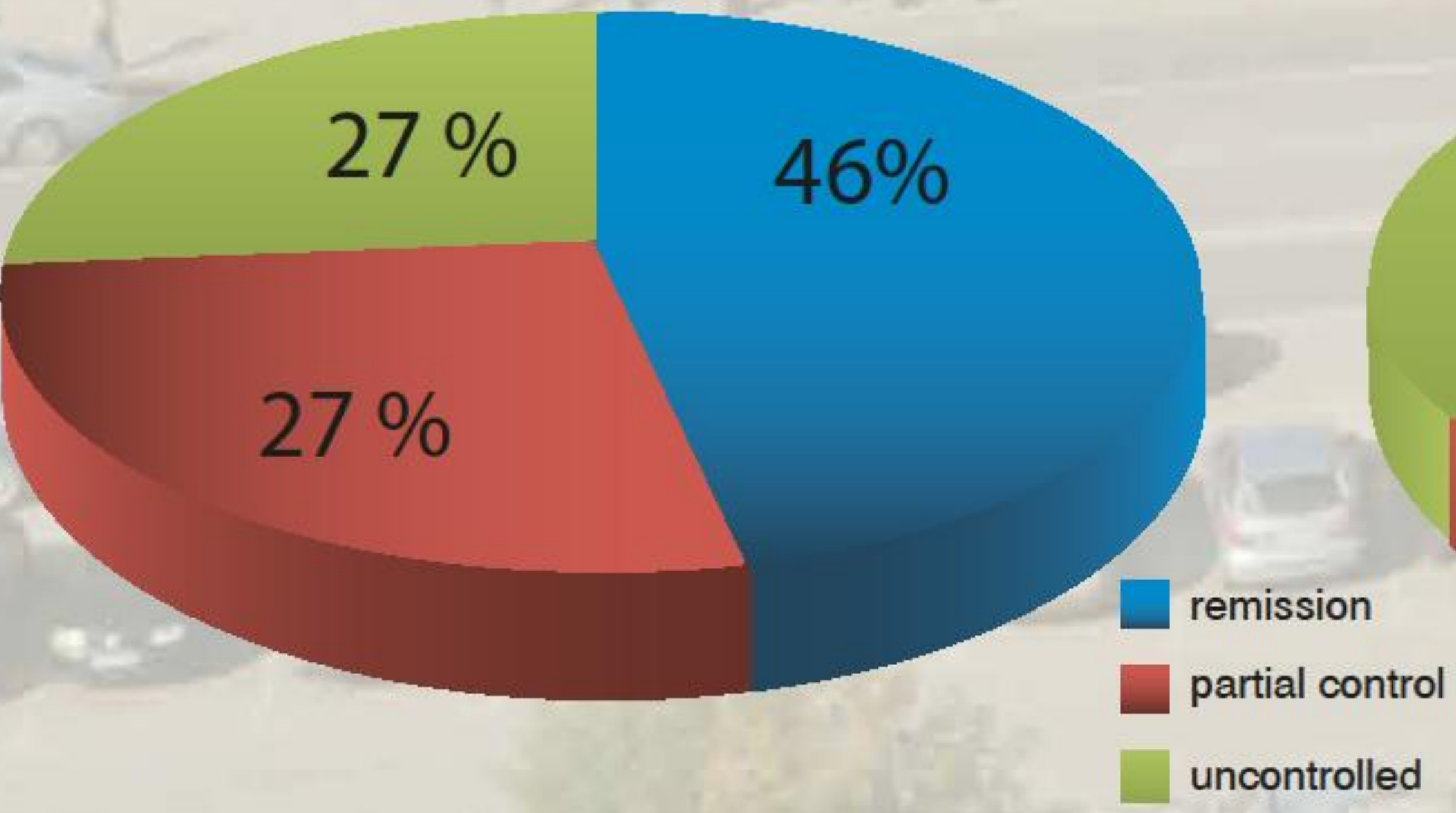

Based on the latest GH and IGF-1 results, in the whole study population

the outcomes were: $46.8 \%$ cured or controlled, $29.8 \%$ partially controlled, and $23.4 \%$ uncontrolled (Figure 2).

Figure 2. Disease outcomes in study population Study population

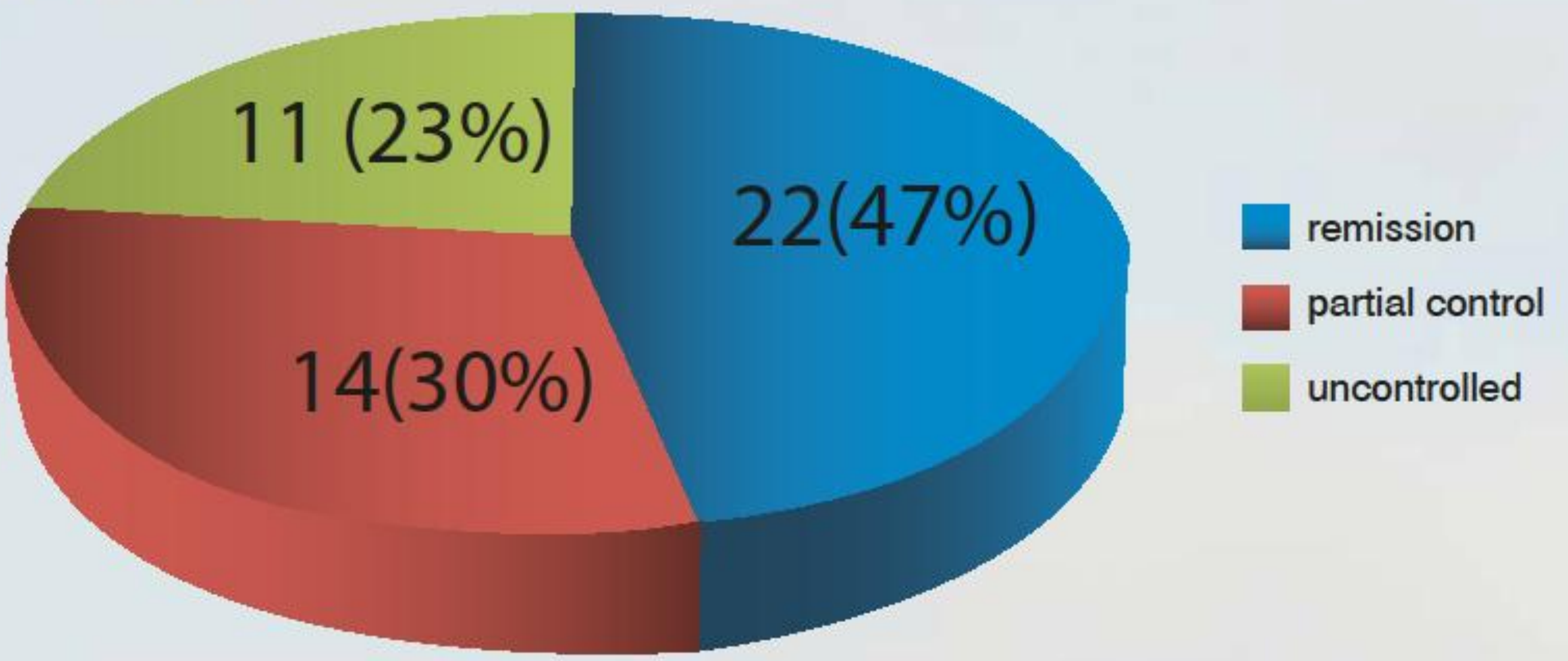

IGF-1 and GH levels in medically treated patients .

Figure 3. Observational period mean IGF-1 in second-line and first-line SSA treatment groups combination treatment groups, $\mathrm{p}=\mathbf{0 . 3 8 4}$

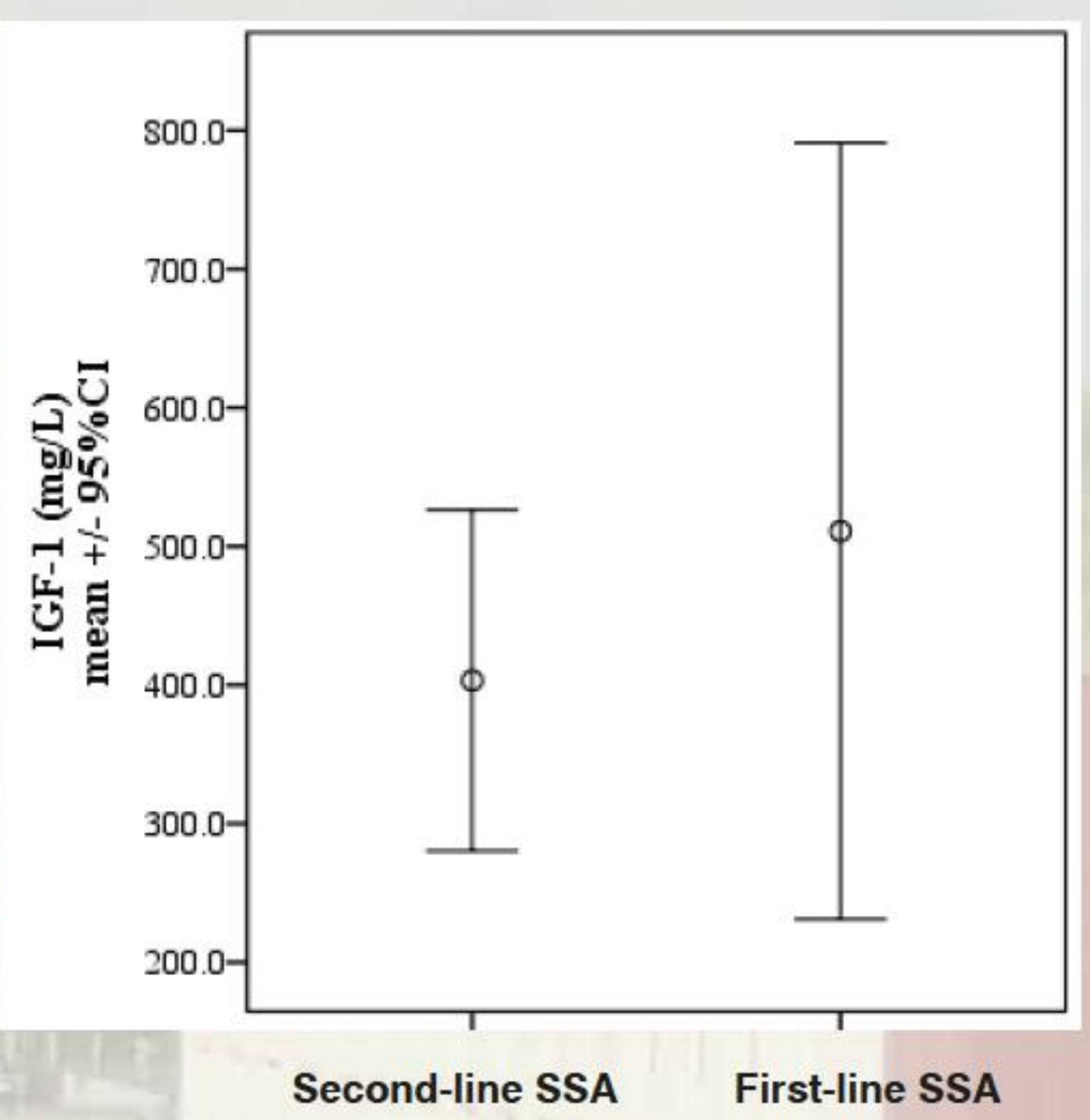

Figure 4. Observational period mean GH in second-line SSA and first-line SSA treatment groups $(P=0.647)$

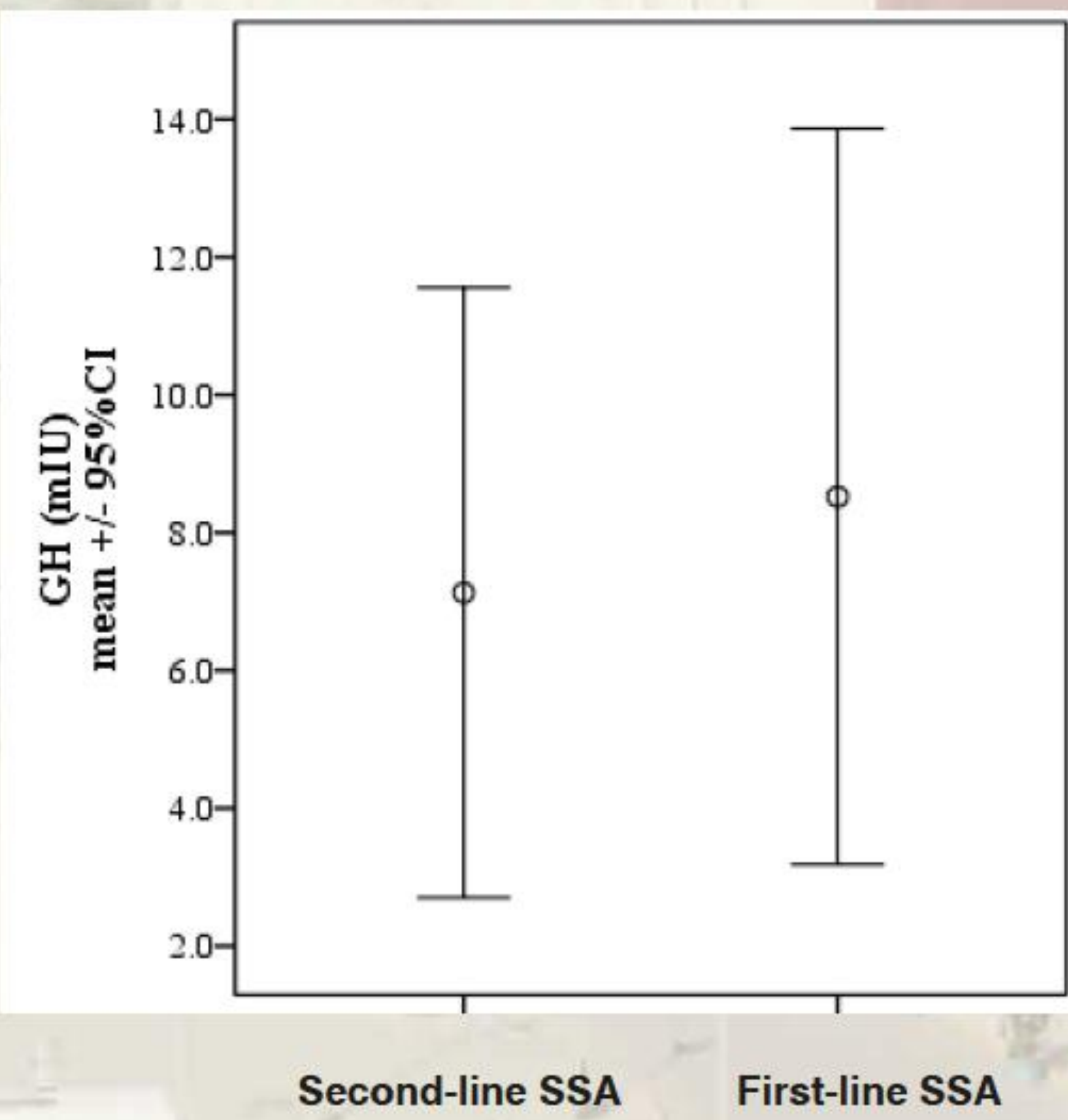

\section{Conclusions:}

CAs disease in a greater percentage of patients. We observed higher observational period IGF-1 and GH levels in first-line SSA treatment patients disease in a greater percentage of patients. We observed higher observational period IGF-1 and GH levels in first-line SSA treatment patients when compared to second-line SSA treathent patents, alhough the differences did not reach statistical significance. Control of the disease remains a challenge despite availability of high-dose SSA treatment as $27-36 \%$ of patients receiving primary or second-line SSA treatment remain uncontrolled.

'Melmed S. Acromegaly pathogenesis and treatment. J Clin Invest 2009; 119:3189-3202.

The -Katznelson L, AlK son JLD, Cook DM et al. Am Diagnosis and Treatment of Acromegaly - 2011 update. Endocr Pract 2011;17 (Suppl 4).

sholdaway IM, Bolland MJ, Gamble GD. A mela-analysis of the efrect of lowening senum lovels of GH and IGF-I on mortality in acromegaly. Eur J Endocrinol 2008;159:89-95

'Katznelson L, Laws ER, Melmed $S$ et al. Acromegaly: an Endocrine Society Clinical Practice Guideline. J Clin Endocrinol Metab 2014;99:3933-3951.

sGiustina A, Chanson P, Kleinberg D et al. A conse 